\title{
Spectral Coexistence in Radar using Xampling
}

\author{
Deborah Cohen, Kumar Vijay Mishra, and Yonina C. Eldar
}

\begin{abstract}
This paper presents a spectrum sharing technology enabling interference-free operation of a surveillance radar and communication transmissions over a common spectrum. A cognitive radio (CRo) receiver senses the spectrum using very low sampling and processing rates. The radar is also modeled as a cognitive system that employs a Xampling-based receiver and transmits in several narrow bands. Our main contribution is the alliance of two previous ideas, CRo and cognitive radar (CRr), and their adaptation to solve the spectrum sharing problem. Finally, we present a hardware realization of our system and demonstrate the SPEctral Coexistence via Xampling (SpeCX) technology through real-time experiments.
\end{abstract}

Keywords-spectrum sharing, cognitive radar, cognitive radio, Xampling, SSPARC

\section{INTRODUCTION}

The unhindered operation of a radar sharing its spectrum with communication ("comm", hereafter) systems has captured a great deal of attention within the operational radar community in recent years [1]. The interest in such spectrum sharing radars is largely due to electromagnetic spectrum being a scarce resource and almost all services having a need for a greater access to the spectrum. With the allocation of available spectrum to newer comm technologies, the radio-frequency $(\mathrm{RF})$ interference in radar bands is on the rise. Spectrum sharing radars aim to use the information from coexisting wireless and navigation services to manage this interference.

Recent research in spectrum sharing radars has focused on $\mathrm{S}$ and C-bands, where the spectrum has seen increasing cohabitation by Long Term-Evolution (LTE) cellular/wireless commercial comm systems. However, spectral interference in some other radar bands existed even before the advent of mobile comm technology (see e.g. [1]). Many synergistic efforts by major agencies are underway for efficient radio spectrum utilization. A significant recent development is the announcement of the Shared Spectrum Access for Radar and Comm (SSPARC) program [2] by the Defense Advanced Research Projects Agency (DARPA). This program is focused on S-band military radars and views spectrum sharing as a cooperative arrangement where the radar and comm services actively exchange information and do not ignore each other. It defines spectral coexistence as equipping existing radar systems with spectrum sharing capabilities and spectral co-design as developing new systems that utilize opportunistic access to the spectrum [3].

A variety of system architectures have been proposed for spectrum sharing radars. Most put emphasis on optimizing the performance of either radar or comm while ignoring the performance of the other. The radar-centric architectures usually assume fixed interference levels from comm and design the system for high probability of detection. Similarly, comm-centric systems attempt to improve performance metrics like the error vector magnitude (EVM) and bit/symbol error

This project has received funding from the European Union's Horizon 2020 research and innovation programme under grant agreement No. 646804-ERC-COG-BNYQ. D.C. is grateful to the Azrieli Foundation for the award of an Azrieli Fellowship. K.V.M. acknowledges partial support via Andrew and Erna Finci Viterbi Fellowship. rate (BER/SER) for interference from radar. With the introduction of the SSPARC program, joint radar-comm performance is being investigated [4]. Real-time exchange of information between radar and comm hardware has not yet been integrated into most system architectures. Our proposed method, described below, incorporates handshaking of spectral information between the two systems.

In this work, we propose a waveform design and receiver processing solution for spectral coexistence of radar-comm systems based on the recently proposed Xampling ("compressed sampling") framework [5]. Xampling is a system architecture that samples and processes analog inputs at rates far below Nyquist, whose underlying structure can be modeled as a union of subspaces (UoS). The Xampling framework performs two main functions: low rate analog to digital conversion (ADC), in which the input is compressed in the analog domain prior to sampling with commercial devices, and low rate digital signal processing, in which the input subspace is detected prior to digital processing. The resulting sparse recovery is performed using compressed sensing (CS) [6] techniques adapted to the analog setting. This concept has been applied to both comm [7] and radar [8].

In our approach, the comm receiver consists of a cognitive radio (CRo) that blind-senses the spectrum from sub-Nyquist samples and provides the radar with spectral occupancy information. Equipped with this spectral map as well as a known radar environment map (REM) detailing typical interference with respect to frequency, the radar transmitter chooses narrow frequency subbands that minimizes interference for its transmission. The receiver samples and processes only these subbands. This technology is adapted from the cognitive radar (CRr) concept explained and practically demonstrated in [9]. By exploiting the sparsity of the target scene, this approach achieves the same range resolution as a conventional wideband signal while transmitting and processing only a few narrow spectral bands. The combined CRo-CRr system results in spectral coexistence via Xampling (SpeCX). This solution has several advantages. It optimizes the radar's performance without interfering with existing comm transmissions. Unlike conventional spectrum sharing radar, the SpeCX radar preserves range resolution despite using less bandwidth for transmission. Both CRo and $\mathrm{CRr}$ receivers use very low sampling rates. Finally, the received signal SNR of the radar is enhanced via cognitive transmission since all the radar power is conccentrated within the subbands that are processed at the receiver.

In the next section, we formulate the spectrum sharing problem. The blind spectrum sensing at the CRo comm receiver and target detection performed by the CRr are discussed in Section III. The SpeCX hardware prototype is presented in Section IV.

\section{Problem Formulation}

Suppose the set of all frequencies of the available common system spectrum is given by $\mathcal{F}$. The comm and radar systems occupy subsets $\mathcal{F}_{C}$ and $\mathcal{F}_{R}$ of $\mathcal{F}$, respectively. Our goal is to design the radar waveform and its support $\mathcal{F}_{R}$, conditional on the fact that the comm 
occupies frequencies $\mathcal{F}_{C}$. We further assume that $\mathcal{F}_{C}$ itself is unknown to the comm receiver, which has to first detect these frequencies. Once $\mathcal{F}_{C}$ is identified, the comm receiver provides a spectral map of occupied bands to the radar. Equipped with the detected spectral map and known REM, the radar waveform generator then selects the available bands with least interference for its transmission and notifies the radar receiver of its selection. The radar conveys the frequencies $\mathcal{F}_{R}$ to the comm receiver as well, so that it may ignore the radar bands while sensing the spectrum. Using our recovery methods, the radar can achieve delay-Doppler recovery performance similar to that of a radar transmitting over the entire band $\mathcal{F}$ despite using only a fraction of this bandwidth.

\section{A. Multiband Communication Signal}

Let $x_{C}(t)$ be a real-valued continuous-time comm signal, supported on $\mathcal{F}=\left[-1 / 2 T_{\mathrm{Nyq}},+1 / 2 T_{\mathrm{Nyq}}\right]$ and composed of up to $N_{\text {sig }}$ transmit waveforms such that

$$
x_{C}(t)=\sum_{i=1}^{N_{\mathrm{sig}}} s_{i}(t)
$$

Formally, the Fourier transform of $x_{C}(t)$, defined by

$$
X_{C}(f)=\lim _{T \rightarrow \infty} \frac{1}{\sqrt{T}} \int_{-T / 2}^{T / 2} x(t) e^{-j 2 \pi f t} \mathrm{~d} t
$$

is zero for every $f \notin \mathcal{F}$. We denote by $f_{\mathrm{Nyq}}=1 / T_{\mathrm{Nyq}}$ the Nyquist rate of $x_{C}(t)$. The waveforms, respective carrier frequencies and bandwidths are unknown. We only assume that the single-sided bandwidth $B_{c}^{i}$ for the $i$ th transmission does not exceed an upper limit $B$, namely $B_{c}^{i} \leq B$ for all $1 \leq i \leq N_{\text {sig. Such sparse wideband signals }}$ belong to the so-called multiband signal model [7]. Let $\mathcal{F}_{C} \subset \mathcal{F}$ be the unknown support of $x_{C}(t)$, where

$$
\mathcal{F}_{C}=\left\{f \| f-f_{i} \mid<B_{c}^{i} / 2 \text {, for all } 1 \leq i \leq N_{\text {sig }}\right\} .
$$

The goal of the comm receiver is to retrieve $\mathcal{F}_{C}$, while sampling and processing $x_{C}(t)$ at low rates to reduce system cost and resources.

\section{B. Pulse Doppler Radar}

A standard pulse Doppler radar transmits a pulse train

$$
r_{T_{X}}(t)=\sum_{p=0}^{P-1} h(t-p \tau), \quad 0 \leq t \leq P \tau,
$$

consisting of $P$ uniformly spaced known pulses $h(t)$. The interpulse transmit delay $\tau$ is the pulse repetition interval (PRI) (or "fast time"); its reciprocal being the pulse repetition frequency (PRF). The entire duration of $P$ pulses in (4) is known as the coherent processing interval (CPI) (or "slow time").

Assume that the radar target scene consists of $L$ non-fluctuating point-targets, according to the Swerling-0 target model [10]. The transmit signal is reflected back by the $L$ targets and these echoes are received by the radar. The radar processor aims at recovering the following information about any of the $L$ targets from the received signal: range-time delay $\tau_{l}$; Doppler frequency $v_{l}$; and complex reflectivity $\alpha_{l}$. The target locations are defined with respect to the polar coordinate system of the radar and their range and Doppler are assumed to lie in the unambiguous time-frequency region. The received signal can then be written as

$$
r_{R_{X}}(t)=\sum_{p=0}^{P-1} \sum_{l=0}^{L-1} \alpha_{l} h\left(t-\tau_{l}-p \tau\right) e^{-j v_{l} p \tau}, \quad 0 \leq t \leq P \tau .
$$

It will be convenient to express $r_{R_{X}}(t)$ as a sum of single frames

$$
r_{R_{X}}(t)=\sum_{p=0}^{P-1} r_{R_{X}}^{p}(t)
$$

where

$$
r_{R_{X}}^{p}(t)=\sum_{l=0}^{L-1} \alpha_{l} h\left(t-\tau_{l}-p \tau\right) e^{-j v_{l} p \tau}, \quad 0 \leq t \leq P \tau,
$$

is the return signal from the $p$ th pulse. In a conventional pulse Doppler radar, the pulse $h(t)=h_{\mathrm{Nyq}}(t)$ is a time-limited baseband function whose Fourier transform is $H_{\mathrm{Nyq}}(f)=\int_{-\infty}^{\infty} h_{\mathrm{Nyq}}(t) e^{-j 2 \pi f t} \mathrm{~d} t$. It is assumed that most of the signal's energy lies within the frequencies $\pm B_{h} / 2$, where $B_{h}$ denotes the effective signal bandwidth. A classical radar signal processor samples each incoming frame $r_{R_{X}}^{p}(t)$ at the Nyquist rate $B_{h}$ to yield the digitized samples $r_{R_{X}}^{p}[n], 0 \leq n \leq N-1$, where $N=\tau B_{h}$. The signal enhancement process employs a matched filter for the sampled frames $r_{R_{X}}^{p}[n]$. This is then followed by Doppler processing where a $P$-point discrete Fourier transform (DFT) is performed on slow time samples. By stacking all the $N$ DFT vectors together, a delay-Doppler map is obtained for the target scene. Finally, the time delays $\tau_{l}$ and Doppler shifts $v_{l}$ of the targets are found on this map using a constant false-alarm rate (CFAR) detector.

Broad bandwidth is necessary to obtain high range resolution, but such a spectral requirement is at odds with the coexisting comm. We, therefore, propose an alternative efficient spectral utilization method wherein the radar transmits several narrow frequency bands instead of a full-band radar signal. In particular, we propose exploiting only a fraction of the bandwidth $B_{h}$ for both transmission and reception of the radar signal, without degrading its range resolution. In SpeCX, the radar transmits a pulse $h(t)$ supported over $N_{b}$ disjoint frequency bands, with bandwidths $\left\{B_{r}^{i}\right\}_{i=1}^{N_{b}}$ centered around the respective frequencies $\left\{f_{r}^{i}\right\}_{i=1}^{N_{b}}$, such that $\sum_{i=1}^{N_{b}} B_{r}^{i}<B_{h}$. The number of bands $N_{b}$ is known to the receiver and does not change during the operation of the radar. The location and extent of the bands $B_{r}^{i}$ and $f_{r}^{i}$ are determined by the radar transmitter through an optimization procedure to identify the least contaminated bands. The resulting radar transmit signal can be written as

$$
H_{R}(f)= \begin{cases}\beta_{i} H_{\mathrm{Nyq}}(f), & f \in \mathcal{F}_{R}^{i}, \text { for } 1 \leq i \leq N_{b}, \\ 0, & \text { otherwise, }\end{cases}
$$

where $\mathcal{F}_{R}^{i}=\left[f_{r}^{i}-B_{r}^{i} / 2, f_{r}^{i}+B_{r}^{i} / 2\right]$ is the set of frequencies in the $i$ th band. The parameters $\beta_{i}>1$ are chosen such that the total transmit power $P_{T}$ of the spectrum sharing radar waveforms remains the same as that of the conventional radar [11]. Denote by

$$
\mathcal{F}_{R}=\bigcup_{i=1}^{N_{b}} \mathcal{F}_{R}^{i}
$$

the radar bandwidth.

\section{Comm Signal Recovery and Radar Target Detection}

We now describe sampling and processing of both comm and radar signals. The shared information between the comm and radar components of SpeCX on the occupied comm support and selected radar spectral bands ensures coexistence between both systems.

\section{A. Cognitive Radio}

The input signal at the comm receiver of the SpeCX system is given by

$$
x(t)=x_{C}(t)+x_{R}(t)
$$


where $x_{R}(t)=r_{T_{X}}(t)+r_{R_{X}}(t)$ is the radar signal sensed by the comm receiver, composed of the transmitted and received radar signals defined in (4) and (5), respectively. Since the frequency support of $x_{C}(t)$ is unknown, a classic processor would sample such a signal at its Nyquist rate, which can be prohibitively high. In this work, we instead use the modulated wideband converter (MWC) [7] - a sub-Nyquist sampling technique that achieves the lower sampling rate bound for perfect blind recovery of multiband signals, namely twice the Landau rate, and is also practically feasible. The MWC is composed of $M$ parallel channels. In each channel, an analog mixing front-end, where $x_{C}(t)$ is multiplied by a mixing function $p_{i}(t)$, aliases the spectrum, such that each band appears in baseband. The mixing functions $p_{i}(t)$ are periodic with period $T_{p}$ such that $f_{p}=1 / T_{p} \geq B$ and have thus the following Fourier expansion:

$$
p_{i}(t)=\sum_{l=-\infty}^{\infty} c_{i l} e^{j \frac{2 \pi}{T_{p}} l t} .
$$

In each channel, the signal next goes through a lowpass filter (LPF) with cut-off frequency $f_{s} / 2$ and is sampled at the rate $f_{s} \geq f_{p}$, resulting in the samples $z_{i}[n]$. Define

$$
N=2\left\lceil\frac{f_{\mathrm{Nyq}}+f_{s}}{2 f_{p}}\right\rceil,
$$

and $\mathcal{F}_{s}=\left[-f_{s} / 2, f_{s} / 2\right]$. Following the calculations in [7], the relation between the known discrete time Fourier transform (DTFT) of the samples $z_{i}[n]$ and the unknown $X_{C}(f)$ is given by

$$
\mathbf{z}(f)=\mathbf{A}\left(\mathbf{x}_{C}(f)+\mathbf{x}_{R}(f)\right), \quad f \in \mathcal{F}_{s},
$$

where $\mathbf{z}(f)$ is a vector of length $M$ with $i$ th element $z_{i}(f)=Z_{i}\left(e^{j 2 \pi f T_{s}}\right)$ and the unknown vector $\mathbf{x}_{C}(f)$ is given by

$$
\mathbf{x}_{C i}(f)=X_{C}\left(f+(i-\lceil N / 2\rceil) f_{p}\right), \quad f \in \mathcal{F}_{s},
$$

for $1 \leq i \leq N$. The vector $\mathbf{x}_{R i}(f)$ is defined similarly. The $M \times N$ matrix $\mathbf{A}$ contains the known coefficients $c_{i l}$ such that $\mathbf{A}_{i l}=c_{i,-l}=c_{i l}^{*}$.

The CRo's goal is now to recover the support of $\mathbf{x}_{C}(f)$ from the low rate samples $\mathbf{z}(f)$. The recovery of $\mathbf{x}_{C}(f)$ for each $f$ independently is inefficient and not robust to noise. Instead, the support recovery paradigm from [7] exploits the fact that the bands occupy continuous spectral intervals so that $\mathbf{x}_{C}(f)$ are jointly sparse for $f \in \mathcal{F}_{p}$. The continuous to finite (CTF) block [7] then produces a finite system of equations, called multiple measurement vectors (MMV) from the infinite number of linear systems (13).

From (13), we have

$$
\mathbf{Q}=\boldsymbol{\Phi} \mathbf{Z} \boldsymbol{\Phi}^{H},
$$

where

$$
\mathbf{Q}=\int_{f \in \mathcal{F}_{p}} \mathbf{z}(f) \mathbf{z}^{H}(f) \mathrm{d} f, \quad \mathbf{Z}=\int_{f \in \mathcal{F}_{p}} \mathbf{x}(f) \mathbf{x}^{H}(f) \mathrm{d} f,
$$

are $M \times M$ and $N \times N$ matrices, respectively. Here, $\mathbf{x}(f)=\mathbf{x}_{C}(f)+\mathbf{x}_{R}(f)$. The matrix $\mathbf{Q}$ is then decomposed to a frame $\mathbf{V}$ such that $\mathbf{Q}=\mathbf{V} \mathbf{V}^{H}$. Clearly, there are many possible ways to select $\mathbf{V}$. One possibility is to construct it by performing an eigendecomposition of $\mathbf{Q}$ and choosing $\mathbf{V}$ as the matrix of eigenvectors corresponding to the non zero eigenvalues. The finite dimensional MMV system is given by

$$
\mathbf{V}=\mathbf{A}\left(\mathbf{U}_{C}+\mathbf{U}_{R}\right) .
$$

The support of the unique sparsest solution of (17) is the same as the support of our original set of equations (13) [7]. Therefore, the support of $\mathbf{U}_{C}$ and $\mathbf{U}_{R}$ are disjoint.
The frequency support $\mathcal{F}_{R}$ of $x_{R}(t)$, given by (9), is known at the comm receiver. From $\mathcal{F}_{R}$, we derive the support $S_{R}$ of the radar slices $\mathbf{x}_{R}(f)$, which is identical to the support of $\mathbf{U}_{R}$, such that

$$
S_{R}=\left\{n\left|n-\frac{f_{R}^{i}}{f_{p}}-\lceil N / 2\rceil\right|<\frac{f_{s}+B_{R}^{i}}{2 f_{p}}\right\},
$$

for $1 \leq i \leq N_{b}$. Our goal can then be stated as recovering the support of $\mathbf{U}_{C}$ from $\mathbf{V}$, given the known support $S_{R}$ of $\mathbf{U}_{R}$. This can be formulated as a sparse recovery with partial support knowledge, studied under the framework of modified CS [12]. The modified-CS idea has been used to adapt CS recovery algorithms to exploit the partial known support a priori information. In particular, greedy algorithms, such as orthogonal matching pursuit (OMP), have been modified to OMP with partial known support (OMP-PKS) [13]. Instead of starting with an initial empty support set, one starts with $S_{R}$ as being the initial support set. In the first iteration, we compute the estimate

$$
\hat{\mathbf{U}}_{1}^{S_{R}}=\mathbf{A}_{S_{R}}^{\dagger} \mathbf{V}, \quad \hat{\mathbf{U}}_{1_{i}}=\mathbf{0}, \quad \forall i \notin S_{R},
$$

and residual

$$
\mathbf{V}_{1}=\mathbf{V}-\mathbf{A}_{S_{R}} \hat{\mathbf{U}}_{1}
$$

The remainder of the algorithm is then identical to OMP.

Once the overall support $S_{C} \cup S_{R}$ is known,

$$
\begin{aligned}
\hat{\mathbf{x}}^{S_{C} \cup S_{R}}[n] & =\mathbf{A}_{S_{C} \cup S_{R}}^{\dagger} \mathbf{z}[n], \\
\hat{\mathbf{x}}_{i}[n] & =0, \quad \forall i \notin S_{C} \bigcup S_{R} .
\end{aligned}
$$

Here, $\mathbf{x}^{S_{C} \cup S_{R}}(f)$ denotes the vector $\mathbf{x}(f)$ reduced to its support, $\mathbf{A}_{S_{C} \cup S_{R}}$ is composed of the columns of $\mathbf{A}$ indexed by $S_{C} \cup S_{R}$ and $\dagger$ is the Moore-Penrose pseudo-inverse. The occupied comm support is then

$$
\mathcal{F}_{C}=\left\{f|| f-(i+\lceil N / 2\rceil) f_{p} \mid \leq \frac{f_{p}}{2}, \text { for all } i \in S_{C}\right\}
$$

\section{B. Cognitive Radar}

The objective of the radar is to identify an appropriate transmit frequency set $\mathcal{F}_{R} \subset \mathcal{F} \backslash \mathcal{F}_{C}$ such that the radar's probability of detection $P_{d}$ is maximized. For a fixed $P_{\mathrm{fa}}$, the $P_{d}$ increases with higher signal to interference and noise ratio (SINR) [14]. Hence, the frequency selection process can, alternatively, choose to maximize the SINR or minimize the spectral power in the undesired parts of the spectrum. The REM is assumed to be known to the radar transmitter in the form of typical interfering energy levels with respect to frequency bands, represented by a vector $\mathbf{y} \in \mathbb{R}^{q}$, where $q$ is the number of frequency bands with bandwidth $b_{y} \triangleq|\mathcal{F}| / q$. In addition, the information available from the CRo indicates that the radar waveform must avoid all the frequencies in the set $\mathcal{F}_{C}$. The goal is now to select subbands from the set $\mathcal{F} \backslash \mathcal{F}_{C}$ with minimal interference energy. We thus seek a block-sparse frequency vector $\mathbf{w} \in \mathbb{R}^{p}$ with unknown block lengths, where $p$ is the number of discretized frequencies, and whose support provides frequency bands with low interference for the radar transmission. Each entry of $\mathbf{w}$ represents a frequency subband of bandwidth $b_{w} \triangleq|\mathcal{F}| / p$. To this end, we adopt the structured sparsity framework from [15] and use the structured greedy algorithm, structured OMP (StructOMP) [15].

At the receiver of this spectrum sharing radar, we employ the sub-Nyquist approach described in [8], where the delay-Doppler map is recovered from the subset of Fourier coefficients defined by $\mathcal{F}_{R}$. 
To this end, we use the structured sparsity framework from [15] that extends standard sparsity regularization to structured sparsity. We adopt the one-dimensional graph sparsity structure whose nodes are the ordered entries of $\mathbf{w}$, so that neighbor nodes are indexed by adjacent frequency bands. The graph dimension is therefore the frequency and its size is $p$. Block sparsity may be enforced by allowing the graph to contain connected regions. In contrast to traditional block sparsity approaches [30], this formulation does not require a priori knowledge on the location of the non-zero blocks. This is achieved by replacing the traditional sparse recovery $\ell_{0}$ constraint by a more general term $c(\mathbf{w})$, referred to as the coding complexity, such that

$$
c(\mathbf{w})=\min \{c(F) \mid \operatorname{supp}(\mathbf{w}) \subset F\},
$$

where $F \subset\{1, \ldots, p\}$ is a sparse subset of the index set of the coefficients of $\mathbf{w}$. In particular, for graph sparsity, the choice of $c(F)$ is simply

$$
c(F)=g \log p+|F|,
$$

where $g$ is the number of connected regions, or blocks, of $F$. This coding complexity favors blocks within the graph.

The resulting optimization problem for finding the block-sparse frequency vector $\mathbf{w}$ can then be expressed as

$$
\min \left\|\mathbf{y}_{\text {inv }}-\mathbf{D w}\right\|_{2}^{2}+\lambda c(\mathbf{w}),
$$

where $\lambda$ is a regularization parameter and $c(\mathbf{w})$ is defined in (23) with $c(F)$ in (24). Here, $\mathbf{y}_{\text {inv }}$ contains element-wise reciprocals of $\mathbf{y}$, namely $\left(\mathbf{y}_{\text {inv }}\right)_{i}=1 / \mathbf{y}_{i}$, so that small values in $\mathbf{y}_{\text {inv }}$ induce corresponding zero blocks in $\mathbf{w}$, and $\mathbf{D}$ is a $q \times p$ matrix that maps each discrete frequency in $\mathbf{w}$ to the corresponding band in $\mathbf{y}_{\text {inv }}$. That is, the $(i, j)$ th entry of $\mathbf{D}$ is equal to 1 if the $j$ th frequency in $\mathbf{w}$ belongs to the $i$ th band in $\mathbf{y}$; otherwise, it is equal to 0 . If we choose $p=q$, then $\mathbf{D}=\mathbf{I}$ is the $q \times q$ identity matrix. Problem (25) can be solved using StructOMP [15].

In the original StructOMP [15], the stopping criterion is based on additional $a$ priori information on the overall sparsity and number of non-zero blocks. We adopt an alternative stopping criterion, based only on the number of blocks, which is known to be equal to $N_{b}$ in our problem. This leads to $N_{b}$ bands in $\mathcal{F}_{R}$ as dictated by the hardware constraints. In the above, additional requirements of transmit power constraints, range sidelobe levels, and minimum separation between the bands can also be imposed. Once the support $\mathcal{F}_{R}$ is identified, a suitable waveform code can be designed using optimization procedures described by e.g. [16, 17].

We now turn to the radar receiver design and describe how a delay-Doppler map can be recovered from only $N_{b}$ transmitted narrow bands. The radar receiver first filters the transmitted bands supported on $\mathcal{F}_{R}$ and computes the Fourier coefficients of the received signal. Consider the Fourier series representation of the aligned frames $r_{R_{X}}^{p}(t+$ $p \tau)$, with $r_{R_{X}}^{p}(t)$ defined in (7):

$$
c_{p}[k]=\frac{1}{\tau} H[k] \sum_{l=0}^{L-1} \alpha_{l} e^{-j 2 \pi k \tau_{l} / \tau} e^{-j v_{l} p \tau}, \quad k \in \kappa,
$$

where $\kappa=\left\{k=\left\lfloor\frac{f}{f_{\text {Nyq }}} N|| f \in \mathcal{F}_{R}\right\}\right.$. From (26), we see that the unknown parameters $\left\{\alpha_{l}, \tau_{l}, v_{l}\right\}_{l=0}^{L-1}$ are embodied in the Fourier coefficients $c_{p}[k]$. The goal is then to recover these parameters from $c_{p}[k], 0 \leq p \leq P-1$.
We adopt the Doppler focusing approach from [8]. Consider the DFT of the coefficients $c_{p}[k]$ in the slow time domain:

$$
\tilde{\Psi}_{v}[k]=\sum_{p=0}^{P-1} c_{p}[k] e^{j v p \tau}=\frac{1}{\tau} H[k] \sum_{l=0}^{L-1} \alpha_{l} e^{-j 2 \pi k \tau_{l} / \tau} \sum_{p=0}^{P-1} e^{j\left(v-v_{l}\right) p \tau} .
$$

The key to Doppler focusing follows from the approximation:

$$
g\left(v \mid v_{l}\right)=\sum_{p=0}^{P-1} e^{j\left(v-v_{l}\right) p \tau} \approx \begin{cases}P & \left|v-v_{l}\right|<\pi / P \tau \\ 0 & \left|v-v_{l}\right| \geq \pi / P \tau\end{cases}
$$

Denote the normalized focused measurements $\Psi_{v}[k]$ so that

$$
\Psi_{v}[k]=\frac{\tau}{P H[k]} \tilde{\Psi}_{v}[k] .
$$

As in traditional pulse Doppler radar, suppose we limit ourselves to the Nyquist grid so that $\tau_{l} / \tau=r_{l} / N$, where $r_{l}$ is an integer satisfying $0 \leq r_{l} \leq N-1$. Then, in vector form, (29) can be approximated as

$$
\boldsymbol{\Psi}_{v}=\mathbf{F}_{\kappa} \mathbf{x}_{v},
$$

where $\boldsymbol{\Psi}_{v}=\left[\Psi_{v}\left[k_{0}\right] \ldots \Psi_{v}\left[k_{K-1}\right]\right], k_{i} \in \kappa$ for $0 \leq i \leq K-1, \mathbf{F}_{\kappa}$ is composed of the $K$ rows of the $N \times N$ Fourier matrix indexed by $\kappa$ and $\mathbf{x}_{v}$ is a $L$-sparse vector that contains the values $\alpha_{l}$ at the indices $r_{l}$ for the Doppler frequencies $v_{l}$ in the "focus zone", that is $\left|v-v_{l}\right|<\pi / P \tau$. It is convenient to write (30) in matrix form, by vertically concatenating the vectors $\boldsymbol{\Psi}_{v}$, for $v$ on the Nyquist grid, namely $v=-\frac{1}{2 \tau}+\frac{1}{P \tau}$, into the $K \times P$ matrix $\boldsymbol{\Psi}$, as

$$
\boldsymbol{\Psi}=\mathbf{F}_{\kappa} \mathbf{X}
$$

The $P$ equations (30) can be solved simultaneously, where in each iteration, the maximal projection of the observation vectors onto the measurement matrix are retained [8]. The following theorem from [8] derives a necessary condition on the minimal number of samples $K$ and pulses $P$ for perfect recovery in a noiseless environment.

Theorem 1. [8] The minimal number of samples required for perfect recovery of $\left\{\alpha_{l}, \tau_{l}, v_{l}\right\}$ with $L$ targets in a noiseless environment is $4 L^{2}$, with $K \geq 2 L$ and $P \geq 2 L$.

Theorem 1 translates into requirements on the total bandwidth of the transmitted bands, such that

$$
B_{\mathrm{tot}}=N \sum_{i=1}^{N_{b}}\left\lceil\frac{B_{r}^{i}}{B_{h}}\right\rceil \text { geq } 2 L .
$$

The multiband design strategy, besides allowing a dynamic form of the transmitted signal spectrum over only a small portion of the whole bandwidth to enable spectrum sharing, has two additional advantages. First, under the conditions of Theorem 1, the CS reconstruction achieves the same resolution as traditional Nyquist processing over a significantly smaller bandwidth. Second, since we only use narrow bands to transmit, the whole power is concentrated in them. Therefore, the SNR in the sampled bands is improved.

\section{SpeCX Prototype}

The SpeCX prototype, shown in Fig. 1, is composed of a CRo receiver and a CRr transceiver. At the heart of the CRo system lies the proprietary MWC board [18] that implements the sub-Nyquist analog front-end receiver. The card first splits the wideband signal into $M=4$ hardware channels, with an expansion factor of $q=5$, yielding $M q=20$ virtual channels after digital expansion (see [7] for more details on the expansion). In each channel, the signal is then mixed with a periodic sequence $p_{i}(t)$, chosen as truncated 


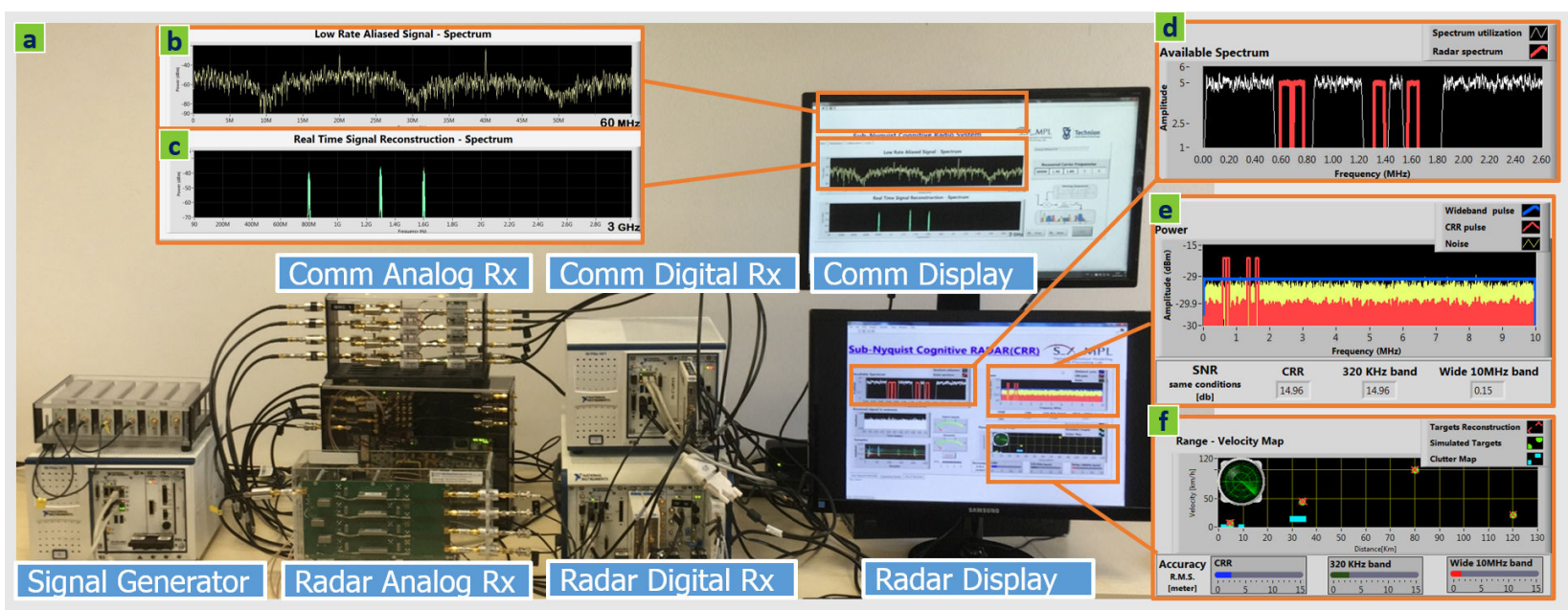

Fig. 1. (a) SpeCX prototype. The system consists of a signal generator, a CRo comm analog receiver including the MWC analog front-end board and the FPGA mixing sequences generator, a comm digital receiver, a CRr analog and receiver. SpeCX comm system display showing (b) low rate samples acquired from one MWC channel at rate $120 \mathrm{MHz}$, and (c) digital reconstruction of the entire spectrum from sub-Nyquist samples. SpeCX radar display showing (d) coexisting comm and CRr, (e) CRr spectrum compared with the full-band radar, and (f) range-velocity display of detected and true locations of the targets.

versions of Gold Codes and generated on a dedicated FPGA, with $f_{p}=20 \mathrm{MHz}$. The sequences are chosen as truncated versions of Gold Codes $[19,20]$, commonly used in telecommunication (CDMA) and satellite navigation (GPS). These were heuristically found to give good detection results in the MWC system [20], primarily due to small bounded cross-correlations within a set. This is useful when multiple devices are broadcasting in the same frequency range. Next, the modulated signal passes through an analog anti-aliasing LPF. Specifically, a Chebyshev LPF of 7th order with a cut-off frequency $(-3 \mathrm{~dB})$ of $50 \mathrm{MHz}$ was chosen for the implementation. Finally, the low rate analog signal is sampled by a National Instruments ${ }^{\odot}$ ADC operating at $f_{s}=(q+1) f_{p}=120 \mathrm{MHz}$ (with intended oversampling), leading to a total sampling rate of $480 \mathrm{MHz}$. The digital receiver is implemented on a National Instruments ${ }^{\circledR}$ PXIe-1065 computer with DC coupled ADC. Since the digital processing is performed at the low rate $120 \mathrm{MHz}$, very low computational load is required in order to achieve real time recovery. MATLAB ${ }^{\circledR}$ and LabVIEW ${ }^{\circledR}$ platforms are used for the various digital recovery operations. The sampling matrix $\mathbf{A}$ is computed only once off-line, using the calibration process outlined in [21].

The prototype is fed with RF signals composed of up to $N_{\text {sig }}=5$ real comm transmissions, namely $K=10$ spectral bands with total bandwidth occupancy of up to $200 \mathrm{MHz}$ and varying support, with Nyquist rate of $6 \mathrm{GHz}$. Specifically, to test the system's support recovery capabilities, an RF input is generated using vector signal generators (VSG), each producing a modulated data channel with individual bandwidth of up to $20 \mathrm{MHz}$, and carrier frequencies ranging from $250 \mathrm{MHz}$ up to $3.1 \mathrm{GHz}$. The input transmissions then go through an RF combiner, resulting in a dynamic multiband input signal, that enables fast carrier switching for each of the bands. This input is specially designed to allow testing the system's ability to rapidly sense the input spectrum and adapt to changes, as required by modern CRo and shared spectrum standards, e.g. in SSPARC program. The system's effective sampling rate, equal to $480 \mathrm{MHz}$, is only $8 \%$ of the Nyquist rate. Support recovery is digitally performed on the low rate samples. The prototype successfully recovers the support of the comm transmitted bands, as demonstrated in Fig. 1(b)-(c). Once the support is recovered, the signal itself can be reconstructed from the sub-Nyquist samples. This step is performed in real-time, reconstructing the signal bands $\mathbf{z}[n]$ one sample at a time. We note that the reconstruction does not require interpolation to the Nyquist rate and the active transmissions are recovered at the low rate of $20 \mathrm{MHz}$, corresponding to the bandwidth of the slices $\mathbf{z}(f)$. The prototype's digital recovery stage is further expanded to support decoding of common comm modulations, including BPSK, QPSK, QAM and OFDM. An example for the decoding of three QPSK modulated bands is shown in Fig. 2. There are no restrictions regarding the modulation type, bandwidth or other signal parameters, since the baseband information is exactly reconstructed regardless of its respective content. By combining both spectrum sensing and signal reconstruction, the MWC prototype serves as two separate comm devices. The first is a state-of-the-art CRo that performs real time spectrum sensing at sub-Nyquist rates, and the second is a unique receiver able to decode multiple data transmissions simultaneously, regardless of their carrier frequencies, while adapting to spectral changes in real time.

The CRr system $[8,9,22]$ includes a custom made sub-Nyquist radar receiver board composed of $N_{b}=4$ parallel channels which sample distinct $N_{b}=4$ bands of the radar signal spectral content. In the $i$ th channel, the transmitted band with center frequency $f_{r}^{i}$ and bandwidth $B_{r}^{i}=80 \mathrm{KHz}$ is filtered, demodulated to baseband and sampled at $250 \mathrm{KHz}$ (with intentional oversampling). This way, four sets of consecutive Fourier coefficients are acquired. More details on the hardware design can be found in [22]. After sampling, the spectrum of each channel output is computed via fast Fourier transform (FFT) and the 320 Fourier coefficients are used for digital recovery of the delay-Doppler map [8]. The prototype simulates transmission of $P=50$ pulses towards $L=10$ targets. The CRr transmits over $N_{b}=4$ bands, $3.2 \%$ of the wideband, after the spectrum sensing process has been completed by the comm receiver. We compare the target detection performance of our CRr with a traditional wideband radar with bandwidth $B_{h}=20 \mathrm{MHz}$. The CRr transmitted bandwidth is thus equal to $3.2 \%$ of the wideband. As shown in Fig. 1(f), the recovery accuracy of

Figure 1 shows windows from the GUI of our CRr system. 


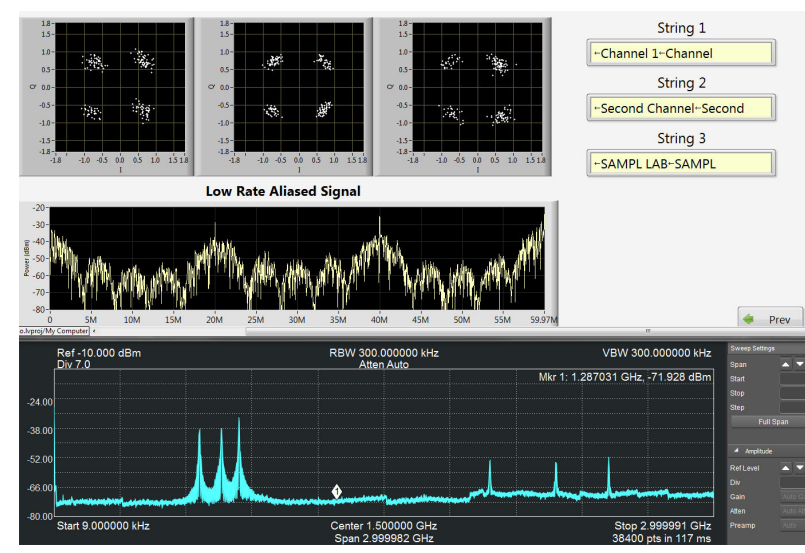

Fig. 2. Demodulation, reconstruction and detection of $N_{\text {sig }}=3$ separate inputs from sub-Nyquist samples using the MWC CR prototype. At the bottom, the input signal is sampled by an external spectrum analyzer showing the entire bandwidth of $3 \mathrm{GHz}$. The sub-Nyquist samples from a MWC channel $z_{i}[n]$ in the Fourier domain are displayed in the middle. The I/Q phase diagrams, showing the modulation pattern of the transmitted bands after reconstruction from the low rate samples, are presented at the top left. In the upper right corner, we can see the actual information that was sent on each carrier, proving that the reconstruction is indeed successful.

Figure 1(d) illustrates the coexistence between the radar transmitted bands in red and the existing comm bands in white. The gain in power is demonstrated in Fig. 1(e); the wideband radar spectrum is shown in blue, our $\mathrm{CRr}$ in red and the noise in yellow in a logarithmic scale. The true and recovered range-velocity maps are shown in Fig. 1(f). All 10 targets are perfectly recovered and clutter, shown in blue, is discarded. Below the map, the range recovery accuracy is shown for 3 scenarios: from left to right, $\mathrm{CRr}$ in blue $(2.5 \mathrm{~m}), 4$ adjacent bands with same bandwidth $(12.5 \mathrm{~m})$ and wideband $(4 \mathrm{~m})$. The poor resolution of the 4 adjacent bands scenario is due to its small aperture. Our CRr system with non-adjacent bands yields better resolution than the traditional wideband scenario.

\section{Summary}

Our SpeCX model proposes a comm and radar spectral coexistence approach through the well-established theory of Xampling. We presented a complete solution that shows recovery of signal in both systems with the minimum of known information about the spectrum. We showed that the SpeCX is practically feasible through the development and real-time testing of our hardware prototype. Some of the other elements of signal model that were not considered in this work include performance of the comm receiver when the radar signal is also contaminated with clutter and hostile jamming.

\section{REFERENCES}

[1] H. Griffiths, L. Cohen, S. Watts, E. Mokole, C. Baker, M. Wicks, and S. Blunt, "Radar spectrum engineering and management: Technical and regulatory issues," Proc. IEEE, vol. 103, no. 1, pp. 85-102, 2015.

[2] G. M. Jacyna, B. Fell, and D. McLemore, "A high-level overview of fundamental limits studies for the DARPA SSPARC program," in IEEE Radar Conf., May 2016.

[3] J. R. Guerci, R. M. Guerci, A. Lackpour, and D. Moskowitz, "Joint design and operation of shared spectrum access for radar and communications," in IEEE Radar Conf., 2015, pp. 761-766.

[4] A. R. Chiriyath, B. Paul, G. M. Jacyna, and D. W. Bliss, "Inner bounds on performance of radar and communications co-existence," IEEE Trans. Signal Process., vol. 64, no. 2, pp. 464-474, 2016.
[5] Y. C. Eldar, Sampling Theory: Beyond Bandlimited Systems. Cambridge University Press, 2015.

[6] Y. C. Eldar and G. Kutyniok, Compressed Sensing: Theory and Applications. Cambridge University Press, 2012.

[7] M. Mishali and Y. C. Eldar, "From theory to practice: Sub-Nyquist sampling of sparse wideband analog signals," IEEE J. Sel. Topics Signal Process., vol. 4, no. 2, pp. 375-391, 2010.

[8] O. Bar-Ilan and Y. C. Eldar, "Sub-Nyquist radar via Doppler focusing," IEEE Trans. Signal Process., vol. 62, pp. 1796-1811, 2014.

[9] D. Cohen, A. Dikopoltsev, R. Ifraimov, and Y. C. Eldar, "Towards sub-Nyquist cognitive radar," in IEEE Radar Conf., 2016.

[10] M. Skolnik, Radar handbook. McGraw-Hill, 1970.

[11] K. V. Mishra and Y. C. Eldar, "Performance of time delay estimation in a cognitive radar," in IEEE Int. Conf. Acoustics, Speech and Signal Process., 2017, to appear.

[12] N. Vaswani and W. Lu, "Modified-cs: Modifying compressive sensing for problems with partially known support," IEEE Trans. Signal Process., vol. 58, no. 9, pp. 4595-4607, 2010.

[13] V. Stankovi, L. Stankovi, and S. Cheng, "Compressive image sampling with side information," in IEEE Int. Conf. Image Process., 2009, pp. 3037-3040.

[14] S. M. Kay, Fundamentals of Statistical Signal Processing, Volume 2: Detection Theory. Prentice Hall, 1998.

[15] J. Huang, T. Zhang, and D. Metaxas, "Learning with structured sparsity," J. Machine Learning Research, vol. 12, pp. 3371-3412, 2011.

[16] A. Aubry, A. De Maio, M. Piezzo, and A. Farina, "Radar waveform design in a spectrally crowded environment via nonconvex quadratic optimization," IEEE Trans. Aerospace and Electronic Syst., vol. 50, no. 2 , pp. $1138-1152,2014$

[17] H. He, P. Stoica, and J. Li, "Waveform design with stopband and correlation constraints for cognitive radar," in Int. Workshop Cognitive Inf. Process., 2010, pp. 344-349.

[18] M. Mishali, Y. C. Eldar, O. Dounaevsky, and E. Shoshan, "Xampling: Analog to digital at sub-Nyquist rates," IET Circuits, Devices $\mathcal{E}$ Syst., vol. 5, pp. 8-20, 2011.

[19] R. Gold, "Optimal binary sequences for spread spectrum multiplexing (Corresp.)," IEEE Trans. Inf. Theory, vol. 13, no. 4, pp. 619-621, 1967.

[20] M. Mishali and Y. C. Eldar, "Expected RIP: Conditioning of the modulated wideband converter," in IEEE Inf. Theory Workshop, Oct 2009, pp. 343-347.

[21] E. Israeli, S. Tsiper, D. Cohen, A. Reysenson, R. Hilgendorf, E. Shoshan, and Y. C. Eldar, "Hardware calibration of the modulated wideband converter," in IEEE Global Commun. Conf., Dec 2014, pp. 948-953.

[22] E. Baransky, G. Itzhak, I. Shmuel, N. Wagner, E. Shoshan, and Y. C. Eldar, "A sub-Nyquist radar prototype: Hardware and applications," IEEE Trans. Aerospace and Electronic Syst., vol. 50, pp. 809-822, 2014. 